\title{
PANDEMIA COVID-19: ¿QUÉ MÁS PUEDO HACER?
}

\author{
COVID-19 PANDEMIC: WHAT ELSE CAN I DO? \\ Lujhon G. Flórez G. 1,2,a
}

En estas últimas semanas marcadas por la pandemia de COVID-19, en Europa se ha popularizado una frase que traducida de su original italiano dice: "Recuerda que a nuestros abuelos se les ordenó ir a la guerra, ja nosotros todo lo que se nos pide es quedarnos en el sillón!".

Aunque científicos de diferentes países y organizaciones están trabajando de manera acelerada en el desarrollo de una vacuna efectiva y en tener fármacos que ayuden en el tratamiento de esta enfermedad, hasta ahora lo mejor que se puede hacer para evitar la propagación acelerada del virus son las medidas higiénicas y de distanciamiento preventivo. Sin embargo, muchos nos preguntamos ¿qué más podemos hacer además de evitar o retrasar la infección? ¿Es posible mejorar nuestras probabilidades de enfrentar exitosamente esta enfermedad?

Dentro de los factores de riesgo para tener un peor pronóstico en caso de infección por el virus SARSCoV-2 se encuentran los siguientes: tabaquismo, edad mayor a 60 años (debido al fenómeno conocido como inmunosenescencia ${ }^{(1)}$, enfermedad cardiovascular, diabetes, hipertensión arterial, enfermedades pulmonares, cáncer y obesidad. Se conoce también que estas enfermedades mencionadas se relacionan entre sí porque comparten un origen común en desarreglos metabólicos a los que subyace la resistencia a insulina (Síndrome Metabólico). Esta resistencia a la acción de la insulina ha sido identificada como un factor condicionante de la respuesta inmunológica necesaria para combatir las infecciones ${ }^{(4)}$.

A continuación, se presentan datos que muestran cómo algunas sencillas elecciones en nuestra vida diaria pueden ayudar a nuestro organismo a estar mejor preparado para resistir o combatir no solamente a las enfermedades crónicas como obesidad, diabetes, enfermedades cardiovasculares, cáncer, demencia, etc., sino también a condiciones agudas como COVID-19:

\section{Decisiones alimentarias}

Aumentar el consumo diario de frutas y verduras ha demostrado inducir mejoría en diferentes aspectos de la función inmunitaria como:

- Proliferación de Linfocitos T y producción de citoquinas inmunoreactivas como Interleucinas 2 y $4^{(5,6)}$ con la ingesta de carotenoides y licopenos, presentes en tomates, pomelos rosas, sandías, ciruelas, así como en otras frutas y vegetales de color rojo o naranja.

- Aumento significativo en la actividad lítica de las células Natural Killer ${ }^{(7)}$ con la ingesta de polifenoles, que se encuentran presentes en forma abundante en múltiples frutas y verduras de coloración rojiza, legumbres como las lentejas, judías, guisantes y soya, cebolla, ajos, pimientos, té y cacao.

- Aumento en más de un 50\% en la concentración de Inmunoglobulina A, con la ingesta de champiñones ${ }^{(8)}$, entre otros vegetales. El aumento en la secreción de lgA disminuye el riesgo de desarrollar infecciones respiratorias virales.

- El consumo de verduras crucíferas, como el brócoli, repollo, kale, facilita la activación de linfocitos intestinales, los cuales a su vez controlan la carga bacteriana y composición del microbiota, relacionando

${ }^{1}$ Clínica de Medicina del Estilo de Vida, Hospital La Carlota, Universidad de Montemorelos, México.

${ }^{2}$ Latin American Lifestyle Medicine Association.

${ }^{a}$ MD, DipIBLM.

Citar como: Lujhon G. Flórez G. Pandemia COVID-19: ¿Qué más puedo hacer?. Rev Rev. Fac. Med. Hum. Abril 2020; 20(2): 175-177. DOI 10.25176/RFMH. v20i2.2941

Journal home page: http://revistas.urp.edu.pe/index.php/RFMH

Artículo publicado por la Revista de la Facultad de Medicina Humana de la Universidad Ricardo Palma. Es un artículo de acceso abierto, distribuído bajo los términos de la Licencia Creative Commons: Creative Commons Attribution 4.0 International, CC BY 4.0 (https://creativecommons.org/licenses/by/4.0/), que permite el uso no comercial, distribución y reproducción en cualquier medio, siempre que la obra original sea debidamente citada. Para uso comercial, por favor póngase en contacto con revista.medicina@urp.pe 
nuestra ingesta alimentaria con la actividad de nuestro sistema inmunológico ${ }^{(9)}$.

- Aumentar la ingesta de fibra mediante el consumo de frutas, verduras, legumbres y cereales integrales induce cambios en el microbiota que favorecen múltiples efectos positivos sobre el sistema inmunológico ${ }^{(10)}$.

- Se recomienda evitar el consumo de carbohidratosy azúcares refinados, debido a que se han relacionado con disminución en la capacidad de respuesta de los leucocitos, tanto en estudios de laboratorio como en estudios in vivo, aumentando así el riesgo de desarrollar infecciones ${ }^{(11,12)}$.

Se recomienda optar por una alimentación variada, basada en plantas, con alimentos integrales lo menos procesados posible, para lograr obtener los conocidos efectos sinérgicos entre los distintos alimentos. Esto es mucho mejor que la búsqueda de "efectos milagrosos" a partir de nutrientes específicos ${ }^{(13)}$.

\section{Actividad física}

Hay evidencia que muestra que las personas que se ejercitan regularmente a una intensidad moderada sufren de menos infecciones que aquellas que son sedentarias $^{(14)}$.

Ejercitarse regularmente ha demostrado tener un impacto favorable sobre el funcionamiento del sistema inmune, llegando incluso a mitigar el efecto de la inmunosenescencia ${ }^{(15)}$, de manera que su práctica es altamente recomendable en todos los grupos etarios. Es necesario aclarar que la actividad física de alto rendimiento se relaciona con disminución en la función inmune, por lo que se recomienda el ejercicio en intensidad moderada.

\section{Sueño y descanso}

El sueño insuficiente aumenta el riesgo de desarrollar enfermedades infecciosas, debido a que altera de diferentes maneras al sistema inmunitario:

- Disminuyendo la respuesta a vacunas ${ }^{(16)}$.

- Aumento de $300 \%$ en la infección por rinovirus en personas que duermen menos de 7 horas en comparación con personas que duermen más de 7 horas por noche ${ }^{(17,18)}$.

- La falta de sueño disminuye la secreción endógena de melatonina lo cual puede tener efectos negativos sobre el sistema inmunológico. Esto debido a que la melatonina puede disminuir la inflamación y lesión pulmonar durante infecciones virales ${ }^{(19)}$, además de ser un estimulador tanto de la inmunidad innata como adaptativa ${ }^{(20)}$
Se recomienda dormir entre 7 y 8 horas cada noche como una de las prácticas fundamentales para poder disfrutar de buena salud.

\section{Manejo del estrés}

Es conocido el efecto negativo del estrés crónico sobre nuestro sistema inmunológico, de tal manera que la tensión agregada por la situación actual puede elevar nuestros niveles de cortisol y disminuir la capacidad de nuestro organismo de luchar contra las infecciones ${ }^{(21)}$.

Si bien es cierto que no podemos "borrar" a la incertidumbre propia de tiempos como los que estamos viviendo, sí que podemos controlar nuestra respuesta al estrés. Además de una alimentación saludable, actividad física moderada y sueño suficiente hay otras medidas que pueden resultar de utilidad como la meditación y los ejercicios de respiración.

\section{Suplementos}

- Vitamina D: Tiene un efecto regulador sobre varios componentes del sistema inmune. Además, su deficiencia ha sido relacionada con un mayor riesgo para severidad de la infección por SARSCoV-2, por lo que se ha propuesto evaluar $y$ suplementar con vitamina $\mathrm{D}$ a personas con riesgo aumentado de contraer la infección o de desarrollar complicaciones ${ }^{(22)}$.

- Vitamina C: Ensayos clínicos han demostrado que la suplementación con Vitamina C puede disminuir la frecuencia, duración y severidad del resfriado común y la incidencia de neumonía(23). Además, se conoce que la vitamina C puede modular la activación de una de las vías implicadas en la "tormenta de citoquinas" involucrada en la severidad de la enfermedad COVID-19(24).

- Zinc: Hay evidencia de que la suplementación con Zinc durante las primeras 24 horas después del inicio de síntomas de resfriado pueden acortar su duración de manera significativa ${ }^{(25)}$, además puede prevenir el ingreso de coronavirus a las células y disminuir su virulencia ${ }^{(26,27)}$. (estos efectos se han estudiado en otros coronavirus distintos al SARSCoV-2). Se desaconseja el uso de suplementos de zinc por vía nasal debido al riesgo de pérdida del olfato, así como la suplementación prolongada con Zinc, debido a sus potenciales efectos adversos neurológicos con el uso prolongado.

Es muy interesante ver que factores como la alimentación, el sueño, la actividad física y la forma de lidiar con el estrés pueden condicionar el riesgo de contagio y en mayor medida la forma como nuestro organismo hace frente a enfermedades agudas como 
son las infecciones. Esperamos que esta editorial pueda ayudar a aquellas personas que motivadas por la crisis actual desean mejorar su estado de salud. Adicionales a las "Medidas Preventivas de Contagio" que han demostrado ser eficaces para contener la transmisión del virus, los hábitos saludables expuestos, juegan un rol a nivel de cada persona, sin caer en una sensación de falsa seguridad.

Seamos agentes de cuidado: de nosotros mismos, de quienes nos rodean y de nuestro entorno.

Correspondencia: Lujhon G. Flórez G.

Dirección: Calle, Fraccionamiento Camino al Vapor \#209, Zambrano, 67512 Montemorelos, N.L., México.

Teléfono: +528262633188

Correo:Iflorez@um.edu.mx

\section{REFERENCIAS BIBLIOGRÁFICAS}

1.Pawelec G., Age and immunity:What is"immunosenescence"?. Experimental Gerontology 105 (2018) 4-9. DOI: 10.1016/j.exger.2017.10.024

2. Reaven G.M. Role of Insulin Resistance in Human Disease. Diabetes (1988) Vol 37, 1595-1607. DOI: 10.2337/diab.37.12.1595

. Crofts C, Zinn C, Wheldon M, Schofield G. Hyperinsulinemia: A unifying theory of chronic disease? Diabesity (2015) 1 (4): 34-43. doi: 10.15562/ diabesity.2015.19

4. Tsai S, Clemente-Casares X, Zhou AC, Lei H, Ahn JJ, Chan YT et al. Insulin Receptor-Mediated Stimulation Boosts T Cell Immunity during Inflammation and Infection. Cell Metab.2018 Dec 4;28(6):922-934.e4. doi: 10.1016/j. cmet.2018.08.003.

5. Watzl B, Bub A, Brandstetter BR, Rechkemmer G. Modulation of human T-lymphocyte functions by the consumption of carotenoid-rich vegetables. $\mathrm{Br}$ J Nutr. 1999 Nov;82(5):383-9. DOI: 10.1017/s0007114599001634

6. Watzl B, Bub A, Briviba K, Rechkemmer G. Supplementation of a low carotenoid diet with tomato or carrot juice modulates immune functions in healthy men. Ann Nutr Metab. 2003;47(6):255-61. DOI: 10.1159/000072397

7. Bub A, Watzl B, Blockhaus $M$, Briviba $K$, Liegibel $U$, Müller $\mathrm{H}$ et al. Fruit juice consumption modulates antioxidative status, immune status and DNA damage. J Nutr Biochem. 2003 Feb;14(2):90-8. DOI: 10.1016/s0955 2863(02)00255-3

8. Jeong SC1, Koyyalamudi SR, Pang G. Dietary intake of Agaricus bisporus white button mushroom accelerates salivary immunoglobulin A secretion in healthy volunteers. Nutrition. 2012 May;28(5):527-31. doi: 10.1016/j. nut.2011.08.005

9. Li Y, Innocentin S, Withers DR, Roberts NA, Gallagher AR, Grigorieva EF et a Exogenous Stimuli Maintain Intraepithelial Lymphocytes via Aryl Hydrocarbon Receptor Activation. Cell (2011) 147, 629-640. Cell. 2011 Oct 28;147(3):629-40. doi: 10.1016/j.cell.2011.09.025

10. Thaiss CA, Zmora N, Levy M, Elinav E. Diet, gut microbiota and immune responses. Nature. 2016 Jul 7;535(7610):65-74. doi: 10.1038/nature18847

11. Sanchez A, Reeser JL, Lau HS, Yahiku PY, Willard RE, McMillan PJ, et al. Role of sugars in human neutrophilic phagocytosis. Am J Clin Nutr. 1973 Nov;26(11):1180-4. DOI: 10.1093/ajcn/26.11.1180

12. Atamna A, Ayada G, Akirov A, Shochat T, Bishara J, Elis A. High blood glucose variability is associated with bacteremia and mortality in patients hospitalized with acute infection. QJM. 2019 Feb 1;112(2):101-106. doi: 10.1093/qjmed/ hcy235

13. Jacobs DR Jr, Gross MD, Tapsell LC. Food synergy: an operational concep for understanding nutrition. Am J Clin Nutr. 2009 May;89(5):1543S-1548S. doi 10.3945/ajcn.2009.26736B.

14. Campbell JP, Turner JE. Debunking the Myth of Exercise-Induced Immune Suppression: Redefining the Impact of Exercise on Immunological Health Across the Lifespan. Front Immunol. 2018 Apr 16;9:648. doi: 10.3389/ fimmu.2018.00648.
15. Duggal NA, Niemiro G, Harridge SDR, Simpson RJ, Lord JM. Can physical activity ameliorate immunosenescence and thereby reduce age-related multimorbidity?. Nat Rev Immunol. 2019 Sep;19(9):563-572. doi: 10.1038/s41577019-0177-9.

16. Taylor DJ, Kelly K, Kohut ML, Song KS. Is Insomnia a Risk Factor for Decreased Influenza Vaccine Response?. Behav Sleep Med. 2017 Jul-Aug:15(4):270-287. doi: 10.1080/15402002.2015.1126596.

17. Cohen S, Doyle WJ, Alper CM, Janicki-Deverts D, Turner RB. Sleep Habits and Susceptibility to the Common Cold. Arch Intern Med. 2009 Jan 12;169(1):62-7. doi: 10.1001/archinternmed.2008.505.

18. Prather AA, Janicki-Deverts D, Hall MH, Cohen S. Behaviorally assessed sleep and susceptibility to the common cold. Sleep. 2015 Sep 1;38(9):1353-9. doi: 10.5665/sleep.4968.

19. Silvestri M1, Rossi GA. Melatonin: its possible role in the management of viral infections-a brief review. Ital J Pediatr. 2013 Oct 3;39:61. doi: 10.1186/18247288-39-61.

20. Boga JA, Coto-Montes A, Rosales-Corral SA, Tan DX, Reiter RJ, Beneficial actions of melatonin in the management of viral infections: a new use for this "molecular handyman"?. Rev Med Virol. 2012 Sep;22(5):323-38. doi: 10.1002/ rmv.1714.

21. Dahbhar FS. Enhancing versus Suppressive Effects of Stress on Immune Function: Implications for Immunoprotection versus Immunopathology. Allergy Asthma Clin Immunol. 2008 Mar 15;4(1):2-11. doi: 10.1186/1710-14924-1-2.

22. William B. Grant, Henry Lahore, Sharon L. McDonnell, Carole A. Baggerly, Christine B. French, Jennifer L. Aliano et al. Evidence that Vitamin D Supplementation Could Reduce Risk of Influenza and COVID-19 Infections and Deaths. Nutrients 2020, 12, 988; doi:10.3390/nu12040988.

23. Hemila $H_{\text {., }}$ Vitamin $\mathrm{C}$ supplementation and respiratory infections: a systematic review. Mil Med. 2004 Nov;169(11):920-5. DOI: 10.7205/ milmed.169.11.920.

24. Choe JY, Kim SK. Quercetin and ascorbic acid suppress fructose - induced NLRP3 inflammasome activation by blocking intracellular shuttling of txnip in human macrophage cell lines. Inflammation. 2017 Jun;40(3):980-994. doi: $10.1007 / \mathrm{s} 10753-017-0542-4$

25. Hemilä H. Zinc lozenges and the common cold: a meta-analysis comparing zinc acetate and zinc gluconate, and the role of zinc dosage. JRSM Open. 2017 May 2;8(5):2054270417694291. doi: 10.1177/2054270417694291.

26. Phillips JM, Gallagher T, Weiss SR. Neurovirulent murine coronavirus JHM SD uses cellular zinc metalloproteases for virus entry and cell-cell fusion. J Virol. 2017 Mar 29;91(8). pii: e01564-16. doi: 10.1128/JVI.01564-16.

27. Han YS, Chang GG, Juo CG, Lee HJ, Yeh SH, Hsu JT et al. Papain-likeprotease 2(PLP2) from severe acute respiratory syndrome coronavirus(SARS-CoV): expression, purification, characterization, and inhibition. Biochemistry. 2005 Aug 2;44(30):10349-59. DOI: 10.1021/bi0504761 